\title{
DJ-1 is involved in the peritoneal metastasis of gastric cancer through activation of the Akt signaling pathway
}

\author{
ZHENG-MING ZHU ${ }^{1 *},{\text { ZHENG-RONG } \text { LI }^{2 *}, \text { YAN HUANG }^{2}, \text { HAI-HONG YU }}^{3}$, \\ XIAO-SHAN HUANG ${ }^{3}$, YU-FENG YAN ${ }^{3}$, JIANG-HUA SHAO ${ }^{1}$ and HE-PING CHEN ${ }^{3}$ \\ ${ }^{1}$ Department of General Surgery, The Second Affiliated Hospital; ${ }^{2}$ Department of General Surgery VI, \\ The First Affiliated Hospital; and ${ }^{3}$ The Key Laboratory of Basic Pharmacology, School of \\ Pharmaceutical Science, Nanchang University, Nanchang 330006, P.R. China
}

Received November 22, 2013; Accepted December 20, 2013

DOI: $10.3892 /$ or.2013.2961

\begin{abstract}
Peritoneal metastasis is a major cause of death in patients with advanced gastric carcinoma. DJ-1 is now considered to play an important role in the metastasis of various malignancies. However, it remains largely unclear whether DJ-1 is involved in the development of peritoneal metastasis by gastric carcinoma. In the present study, we showed that the expression of DJ-1 was significantly upregulated in gastric cancer specimens with peritoneal metastasis compared to those without peritoneal metastasis. Knockdown of DJ-1 expression significantly inhibited invasion and migration, in vitro and the in vivo peritoneal metastatic abilities of SGC7901 gastric cancer cells. Moreover, knockdown of DJ-1 also diminished the expression of matrix metallopeptidase (MMP)-2 and MMP-9. All of these effects were reversed by restoration of DJ-1 expression. Following investigation of the pathway through which DJ-1 regulates cell invasion and migration, DJ-1 was found to cause phosphorylation of Akt in SGC7901 gastric cancer cells. Inhibition of the Akt pathway in SGC7901 cells mimicked the effects of DJ-1 knockdown on cell migration, invasion, MMP-2 and MMP-9 expression, and abolished the effects of DJ-1 in promoting SGC7901 cell invasion and migration. Taken together, the present study revealed that DJ-1 plays an important role in the development of peritoneal carcinomatosis from gastric
\end{abstract}

Correspondence to: Dr Jiang-Hua Shao, Department of General Surgery, The Second Affiliated Hospital, Nanchang University, Nanchang 330006, P.R. China

E-mail: shao5022@163.com

Dr He-Ping Chen, The Key Laboratory of Basic Pharmacology, School of Pharmaceutical Science, Nanchang University, Nanchang 330006, P.R. China

E-mail: chenheping69@hotmail.com

*Contributed equally

Key words: DJ-1, peritoneal metastasis, gastric carcinoma, Akt pathway carcinoma, at least partially through activation of the Akt pathway and consequent upregulation of MMP-2 and MMP-9 expression. Thus, DJ-1 may be a potential therapeutic target for peritoneal carcinomatosis of gastric carcinoma.

\section{Introduction}

Peritoneal metastasis, a common feature of the natural history of gastric cancer, is the most frequent cause of death in patients with advanced gastric carcinoma (1-3). Despite advances in therapeutic modalities for peritoneal diseases such as combination chemotherapy and chemohyperthermia, the 5-year survival rate of patients with peritoneal carcinomatosis is only $2 \%$ (4). Thus, there is an urgent need for further knowledge of the molecular mechanisms responsible for peritoneal metastasis of gastric carcinoma and for identifying potential novel therapeutic targets, which may facilitate the development more effective treatment modalities.

It is now well established that peritoneal metastasis is a multistep process involving the detachment of malignant cells from the primary tumor, their migration into the peritoneal cavity, their attachment to the peritoneum, and finally their proliferation to form secondary tumor foci. Differences in metastatic potential are expected to result from differences in expression of a combination of genes related to cell adhesion, apoptosis, signal transduction and other processes (5). Yet, details of the molecular basis of peritoneal metastasis remain unclear.

DJ-1, a ubiquitously expressed and highly conserved intracellular protein, was originally discovered as a novel oncogene product that can transform mouse NIH3T3 cells in combination with H-Ras or c-Myc (6). Subsequent studies demonstrated that DJ-1 has multiple functions and is involved in diverse cellular processes ranging from cellular transformation, transcriptional regulation, antioxidative stress response to control of male infertility, among several others (7-11). Recently, accumulating evidence has shown that DJ-1 is overexpressed in many types of malignant tumors, including breast (12), primary lung cancer (13), leukemia (14), prostate (15), cervical (16), papillary thyroid (17) and pancreatic cancer (18), indicating a possible role for DJ-1 in the occurrence and development of tumors. Moreover, of note, several 
recent studies have also reported that DJ-1 may be related to cancer metastasis. For example, Pardo et al (19) found that DJ-1 expression was upregulated in uveal melanoma cells that had a high degree of metastatic potential, when compared with that in corresponding normal cells. Yuen et al (20) found that DJ-1 was amplified and overexpressed in esophageal squamous cell carcinoma metastatic lesions when compared with that in normal esophageal epithelium and primary tumors. In addition, DJ-1 expression was found to be significantly correlated with human non-small cell lung cancer lymphatic metastasis (21). DJ-1 was also confirmed to promote in vitro and in vivo invasion and metastasis of human glioma cells and pancreatic cancer cells $(22,23)$. These results suggest that high expression of DJ-1 is a key alteration contributing to the metastasis of tumor cells.

However, although considerable evidence has accumulated suggesting that DJ-1 may play a major role in tumorigenesis and metastasis, virtually nothing is known regarding its involvement in the development of peritoneal metastasis by gastric carcinoma. Therefore, the objective of this study was to investigate the role of DJ-1 in peritoneal metastasis of gastric carcinoma and to determine the underlining mechanisms of this process.

In the present study, we present initial evidence that DJ-1 is highly expressed in gastric cancer with peritoneal metastasis. The existence of DJ-1 in gastric cancer promotes the invasive and metastatic abilities of gastric cancer cells through activation of the Akt pathway and consequent upregulation of MMP-2 and MMP-9 expression.

\section{Materials and methods}

Chemicals and reagents. Dulbecco's modified Eagle's medium (DMEM), fetal bovine serum (FBS) and Lipofectamine 2000 transfection reagent were purchased from Invitrogen (Carlsbad, CA, USA). API-2 (an inhibitor of Akt) was purchased from Sigma Chemical Co. (St. Louis, MO, USA). Transwell inserts were purchased from Corning Incorporated (Corning, NY, USA). Matrigel was purchased from BD Biosciences (Bedford, MA, USA). An anti-DJ-1 antibody was obtained from Abcam (Cambridge, MA, USA). Anti-phospho-Akt (Thr 308) and anti-Akt antibodies were purchased from Cell Signaling Technology (Beverly, MA, USA). MMP-2/MMP-9 inhibitor I, anti-MMP-2, anti-MMP-9 and anti- $\beta$-actin primary antibodies were from Santa Cruz Biotechnology (Santa Cruz, CA, USA). All other chemicals were purchased from Sigma Chemical, unless otherwise noted.

Cell lines and cell culture. Human gastric adenocarcinoma cell lines, SGC7901, MKN45, MKN28 and BGC823, and the normal human gastric mucosal cell line GES-1, were obtained from the Type Culture Collection of the Chinese Academy of Sciences (Shanghai, China). Cells were cultured in DMEM containing $10 \%$ heat-inactivated FBS, $100 \mathrm{U} / \mathrm{ml}$ penicillin and $100 \mu \mathrm{g} / \mathrm{ml}$ streptomycin. All cultures were maintained in a humidified $5 \% \mathrm{CO}_{2}$ atmosphere at $37^{\circ} \mathrm{C}$, and cells were passaged by treating them with $0.02 \%$ EDTA in phosphatebuffered saline (PBS) and $0.25 \%$ trypsin after they had achieved confluence. Exponentially growing cells were used for the experiments.
Tissue samples. Tissue samples of primary tumors were obtained during gastrectomy from untreated patients with gastric carcinomas. Histological diagnosis was confirmed for each specimen. All surgical samples were obtained from the Department of General Surgery, First Affiliated Hospital, Nanchang University (Nanchang, China). A written informed consent for molecular analysis of the surgical sample was obtained from each patient.

Western blot analysis. Total proteins were extracted from the tumor tissues or cells using a protein extraction kit (Pierce, Rockford, IL, USA) according to the manufacturer's protocols. The protein content was determined by the Lowry method using a DC protein assay kit (Bio-Rad Laboratories, Hercules, CA, USA). Equal amounts of proteins were resolved by $10 \%$ SDS-PAGE and transferred to polyvinylidene difluoride membranes. After blocking with 5\% non-fat milk, the blots were then probed with primary antibodies against DJ-1 (dilution 1:2,000), Akt (1:1,000), phospho-Akt (1:1,000), MMP-2 (1:500), MMP-9 (1:500) and $\beta$-actin $(1: 3,000)$. The bound antibodies were detected using appropriate horseradish peroxidase-conjugated secondary antibody and visualized using an enhanced chemiluminescence system (Millipore, Billerica, MA, USA). The levels of each protein were standardized to the loading control, $\beta$-actin, and were quantified using an ImageJ 1.41 program (National Institutes of Health, Bethesda, MD, USA).

Generation of DJ-1-knockdown SGC7901 cells. Three candidate human DJ-1 siRNA target sequences and a negative control siRNA (NC siRNA) sequence were designed using the siRNA Target Finder and Design Tool available at www. ambion.com/techlib/misc/siRNA_finder.html. These siRNA sequences were 5'-AAATGGAGACGGTCATCCCTG-3' (DJ-1 siRNA-1), 5'-AAGACCCAGTACAGTGTAGCC-3' (DJ-1 siRNA-2), 5'-AAGGTCACCGTTGCAGGCCTG-3' (DJ-1 siRNA-3), and 5'-AAGACGCTGAAGACTCTTGGC-3' (NC siRNA), respectively. Subsequently, these siRNA duplexes were synthesized and were transiently transfected into SGC7901 cells according to manufacturer's instructions. Their efficacies in extinguishing DJ-1 expression were evaluated by western blot analysis. DJ-1 siRNA-3, above, showing the highest efficacy, was selected for establishing DJ-1knockdown SGC7901 cell lines. Briefly, the template oligonucleotides corresponding to DJ-1 siRNA-3 and NC siRNA were, respectively, synthesized and ligated into $B b s \mathrm{I}-$ BamHI sites of the pGPU6/GFP/Neo vector (Shanghai Genepharma, Shanghai, China) to generate pGPU6/GFP/ Neo-DJ-1-siRNA and pGPU6/GFP/Neo-NC-siRNA recombinant plasmids. After being confirmed by DNA sequencing, the pGPU6/GFP/Neo-DJ-1-siRNA and pGPU6/GFP/Neo-NCsiRNA vectors were transfected into SGC7901 cells using Lipofectamine 2000 reagent according to the manufacturer's protocol. Twenty-four hours after transfection, cells were split into medium containing G418 (400 $\mu \mathrm{g} / \mathrm{ml})$ (Calbiochem, Nottingham, UK) and maintained for 14 days, with regular replenishment of medium and drug. At this time individual clones were selected, expanded, and screened for expression of DJ-1 by western blot analysis. Clones of the SGC7901 cells stably transfected with either pGPU6/GFP/Neo-DJ-1-siRNA 
A
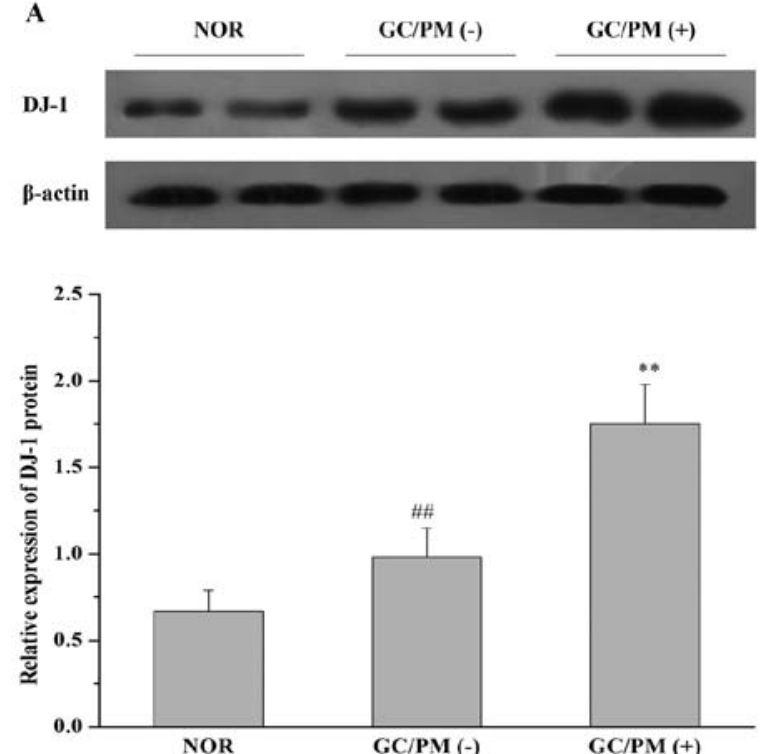

B

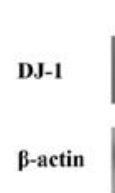

GES-1 $\quad$ BGC823 $\quad$ MKN28 $\quad$ SGC7901 MKN45
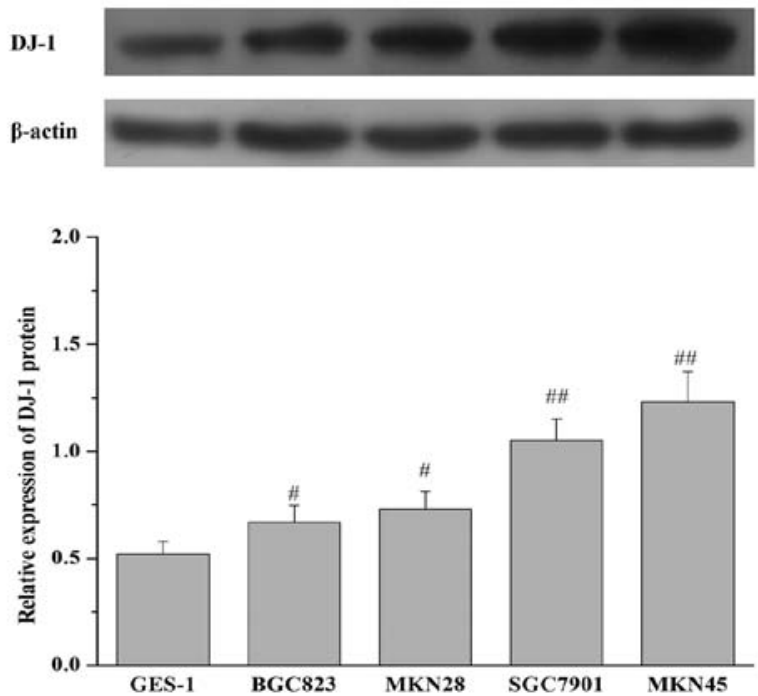

Figure 1. Western blot analysis of DJ-1 protein expression in gastric cancer tissues and cell lines. (A) The expression of DJ-1 protein in 37 specimens of normal gastric mucosa (NOR), 40 specimens of gastric carcinoma without peritoneal metastasis [GC/PM (-)], and 28 specimens of gastric carcinoma with peritoneal metastasis $[\mathrm{GC} / \mathrm{PM}(+)]$ was analyzed by western blot analysis. Relative values for DJ-1 protein were determined as ratios to $\beta$-actin. Each value represents the mean \pm SD. ${ }^{\# /} \mathrm{P}<0.01$ vs. NOR; ${ }^{* *} \mathrm{P}<0.01$ vs. GC/PM (-). (B) The expression of DJ-1 protein in one normal human gastric mucosal cell line (GES-1) and four gastric cancer cell lines (BGC823, MKN28, SGC7901 and MKN45) was analyzed by western blot analysis. $\beta$-actin was used as an internal control ${ }^{\#} \mathrm{P}<0.05,{ }^{\# \#} \mathrm{P}<0.01$, vs. GES-1 cells. A representative blot of each experiment is shown with the densitometric analysis corresponding to the mean \pm SD of four independent experiments.

(designated as SGC7901/DJ-1-siRNA) or pGPU6/GFP/ Neo-NC-siRNA (designated as SGC7901/NC-siRNA) were maintained in DMEM supplemented with $200 \mu \mathrm{g} / \mathrm{ml} \mathrm{G418.}$

Construction of the DJ-1 gene expression vector. For rescue of DJ-1 expression levels, rat DJ-1, containing two mismatches with respect to the human-specific DJ-1 siRNA-3, was introduced in SGC7901/DJ-1-siRNA cells. To obtain the coding region of rat wild-type DJ-1 cDNA, oligonucleotide primers complementary to the 5' and 3 ' ends of DJ-1 were designed that incorporated the restriction sites EcoRI and KpnI: Primer S, 5'-CCGGAATTCAATGGCATCCAAAAGAGC-3'; and Primer A, 5'-CGGGGTACCCTAGTCTTTGAGAACAAG CG-3'. PCR products were digested with EcoRI and KpnI and analyzed by agarose gel electrophoresis. Bands of the expected length were cut out and ligated into the eukaryotic expression vector pFLAG-CMV-4 (Sigma), which had been digested with EcoRI and KpnI. Subsequently, the generated construct was confirmed by sequencing and further referred to as pFLAGrDJ-1.

Analysis of in vitro migration and invasion. Cell migration and invasion assays were performed using $8.0-\mu \mathrm{m}$ pore size Transwell inserts as previously described (24). For all migration assays, in brief, the undersurface of the membrane was coated with fibronectin $(10 \mu \mathrm{g} / \mathrm{ml})$ in PBS at $37^{\circ} \mathrm{C}$ for $2 \mathrm{~h}$. The membrane was washed in PBS to remove excess ligand, and the lower chamber was filled with $0.6 \mathrm{ml}$ DMEM with $10 \% \mathrm{FBS}$. Cells were serum-starved overnight ( $0.5 \%$ FBS $)$, harvested with trypsin/EDTA and washed twice with serum-free DMEM. Then, cells were resuspended in migration medium (DMEM with $0.5 \% \mathrm{FBS}$ ), and $1 \times 10^{5}$ cells in $0.1 \mathrm{ml}$ were added to the upper chamber. After $24 \mathrm{~h}$ at $37^{\circ} \mathrm{C}$, the cells on the upper surface of the membrane were removed using cotton swabs. The migrated cells that had attached to the lower surface were fixed in methanol at room temperature for $30 \mathrm{~min}$ and stained for 20 min with a solution containing $0.5 \%$ crystal violet and $2 \%$ ethanol in $100 \mathrm{mM}$ borate buffer $(\mathrm{pH} \mathrm{9.0)}$. The number of cells that migrated to the lower surface of the membrane were counted under a microscope in random five fields at x100 magnification. For the cell invasion assay, all procedures were carried out as in the migration assay except that Matrigel was used to coated beforehand the upper surface of the chambers according to the manufacturer's protocol.

In vivo peritoneal metastasis assay. Pathogen-free, 6-weekold, female BALB/c nude mice were purchased from Hunan Slack King of Laboratory Animal Co., Ltd. (Changsha, China). All animal experiments complied with the Guidelines for the Care and Use of Laboratory Animals of Nanchang University. The peritoneal metastasis model was conducted as previously described $(25,26)$. In brief, mice weighing $18-22 \mathrm{~g}$ were injected intraperitoneally with $5 \times 10^{6}$ SGC7901 cells in $1 \mathrm{ml}$ PBS. Mice were sacrificed 21 days after SGC7901 cell injection, and the size and the number of metastatic nodules in the peritoneal cavity were counted by the naked eye as previously described (27).

Statistical analysis. The results are presented as means \pm SD. The unpaired t-test was used to compare the differences between groups. Multiple group means were compared by ANOVA followed by LSD post hoc test. Differences with a two-tailed $\mathrm{P}<0.05$ were considered to indicate statistically significant results. 
A
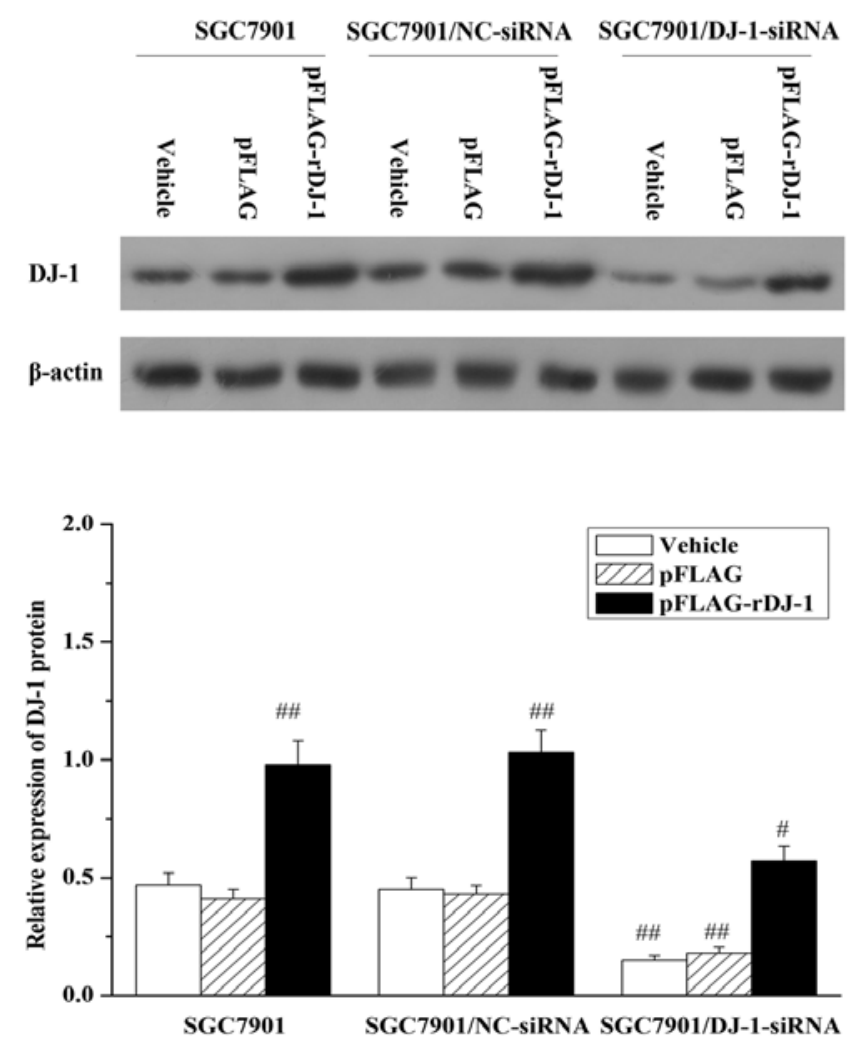

B
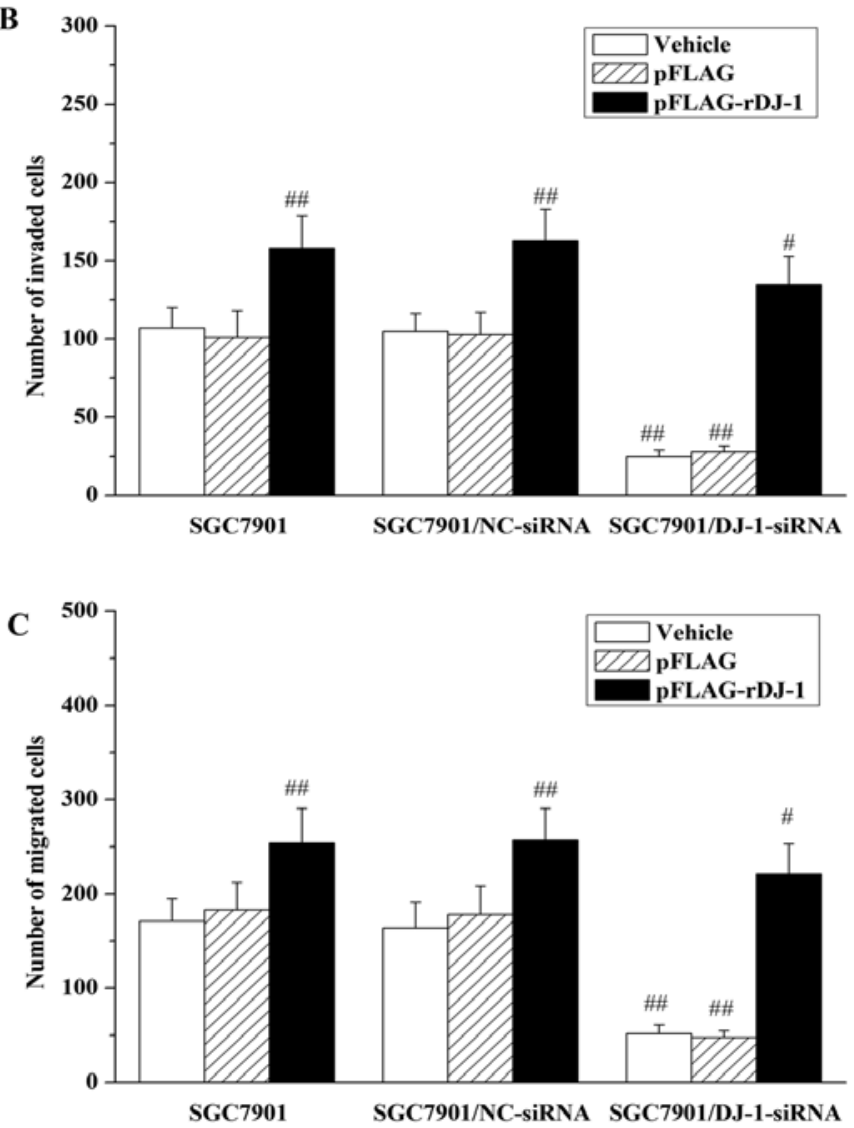

Figure 2. DJ-1 regulates gastric cancer cell migration and invasion. SGC7901, SGC7901/NC-siRNA and SGC7901/DJ-1-siRNA cells were transfected with or without empty vector pFLAG or pFLAG-rDJ-1. Twenty-four hours later, (A) the expression of DJ-1 protein and (B) cell invasion and (C) migration were respectively analyzed, as described in Materials and methods. Each value represents the mean $\pm \mathrm{SD}$ of four independent experiments. ${ }^{\#} \mathrm{P}<0.05$, ${ }^{\# \#} \mathrm{P}<0.01, \mathrm{vs}$. SGC7901 + Vehicle.

\section{Results}

DJ-1 is overexpressed in gastric cancer tissues with peritoneal metastasis and in gastric cancer cell lines with higher metastatic potential. To investigate the relationship between the expression of DJ-1 and peritoneal metastasis of gastric cancer, we first detected the expression of DJ-1 protein in 37 specimens of normal gastric mucosa, 40 specimens of gastric carcinoma without peritoneal metastasis, and 28 specimens of gastric carcinoma with peritoneal metastasis by western blot analysis. The relative values for DJ-1 protein were determined as ratios to $\beta$-actin (Fig. 1A). The relative value for DJ-1 protein in normal gastric mucosa (37 cases, mean $\pm \mathrm{SD}$ ) was $0.67 \pm 0.12$. In the primary tumors of gastric carcinoma without peritoneal metastasis ( 40 cases), the value for DJ-1 was $0.98 \pm 0.17$, whereas in the primary tumors of gastric carcinoma with peritoneal metastasis ( 28 cases), this value was $1.75 \pm 0.23$. The results revealed that the expression of DJ-1 was significantly upregulated in gastric carcinoma with peritoneal metastasis when compared to the expression in gastric carcinoma without peritoneal metastasis or normal gastric mucosa. In addition, we also analyzed the expression of DJ-1 protein in one normal human gastric mucosa cell line GES-1 and four gastric cancer cell lines of different metastatic potential (BGC823, MKN28, SGC7901 and MKN45), of which SGC7901 and MKN45 were demonstrated to have higher metastatic potential compared with BGC823 and MKN28 (28). As shown in Fig. 1B, DJ-1 expression in the four gastric cancer cell lines was much higher than that in the GES-1 cell line. Furthermore, DJ-1 expression was higher in the SGC7901 and MKN45 cells when compared with that in the BGC823 and MKN28 cells. These results suggest that overexpression of DJ-1 may be closely related to the development of peritoneal metastasis by gastric carcinoma.

DJ-1 promotes in vitro invasion and migration and in vivo peritoneal metastatic abilities of gastric cancer cells. Next, to determine whether DJ-1 has a causal function in gastric cancer peritoneal metastasis, we studied the effects of DJ-1 knockdown on gastric cancer cell invasion and migration using SGC7901/DJ-1-siRNA cells. This experiment revealed that DJ-1 expression level in the SGC7901/DJ-1-siRNA cells was significantly reduced (Fig. 2A). More importantly, the knockdown of DJ-1 expression led to a significant reduction in cell invasion (Fig. 2B) and migration (Fig. 2C), as compared to the control cells (SGC7901 or SGC7901/NC-siRNA). To further confirm that the reduced invasion and migration were caused by specific downregulation of DJ-1 protein and were not due to off-target effects, we restored DJ-1 expression in the SGC7901/DJ-1-siRNA cells by transfection of the pFLAG-rDJ-1 vector. As shown in Fig. 2A, after transient transfection of pFLAG-rDJ-1 for $24 \mathrm{~h}$, DJ-1 expression in the SGC7901/DJ-1-siRNA cells was restored to a level higher 


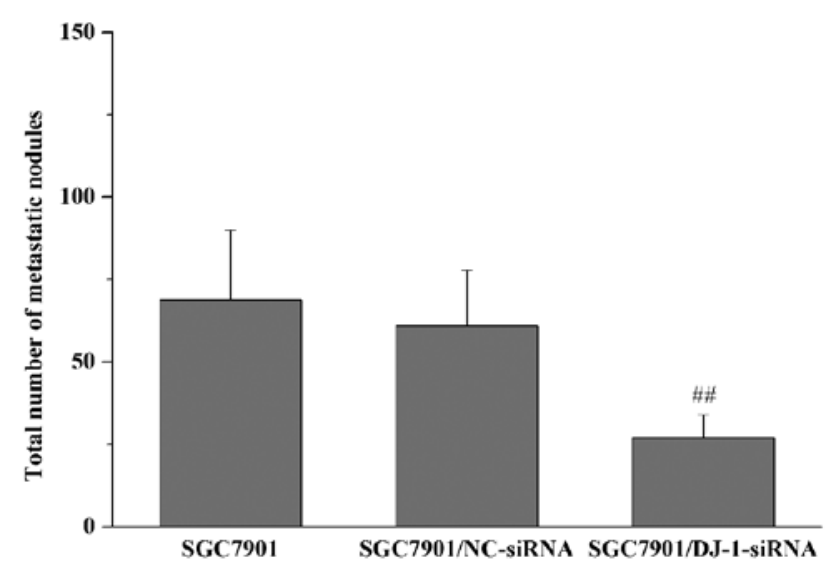

Figure 3. Effects of DJ-1 knockdown on peritoneal metastasis of SGC7901 gastric cancer cells in vivo. SGC7901, SGC7901/NC-siRNA and SGC7901/DJ-1-siRNA cells were intraperitoneally injected into nude mice. Twenty-one days later, mice were sacrificed, and the total number of metastatic nodules in the peritoneal cavity were counted, as described in Materials and methods. Each value represents the mean \pm SD of six independent experiments. ${ }^{\# /} \mathrm{P}<0.01$ vs. the SGC7901 group.

than the baseline level. Notably, the restoration of DJ-1 expression was also accompanied by the restoration of cell invasion (Fig. 2B) and migration (Fig. 2C) in the SGC7901/DJ-1-siRNA cells. In contrast, transfection of pFLAG-rDJ-1 markedly increased DJ-1 levels concomitant with an obvious elevation in cell invasion and migration activities in the parental SGC7901 and SGC7901/NC-siRNA cells.

Subsequently, we examined whether DJ-1 knockdown results in the inhibition of in vivo peritoneal dissemination of gastric cancer cells. Nude mice received i.p. injections of SGC7901, SGC7901/NC-siRNA or SGC7901/DJ-1-siRNA cells. The macroscopic nodules of peritoneal dissemination were then counted 3 weeks after tumor inoculation. The results showed that metastatic nodules developed preferentially around the vessels of the intestinal mesentery. Compared with the control cells (SGC7901 and SGC7901/NC-siRNA cells), i.p. inoculation of SGC7901/DJ-1-siRNA cells led to a significant decrease in the number of metastatic nodules in the peritoneal cavity (Fig. 3). Taken together, these observations suggest that DJ-1 has the potential to promote in vitro invasion and migration and in vivo peritoneal metastasis of gastric cancer cells.

Matrix metallopeptidase (MMP)-2 and MMP-9 are involved in the invasion and migration of gastric cancer cells regulated by $D J-1$. Extracellular matrix degradation is an essential step in tumor invasion and metastasis, which is mainly mediated by MMP-2 and MMP-9 (29). To understand the mechanisms underlying DJ-1 regulation of cell invasion and migration, we further analyzed MMP-2 and MMP-9 expression in the SGC7901/DJ-1-siRNA cells by western blot analysis. As expected, silencing of DJ-1 resulted in reduction in MMP-2 and MMP-9 protein expression in the SGC7901/DJ-1-siRNA cells when compared to these levels in the parental SGC7901 and SGC7901/NC-siRNA cells, which was reversed by restoration of DJ-1 expression (Fig. 4). Additionally, the 24-h transfection of pFLAG-rDJ-1 markedly increased the expression of MMP-2 and MMP-9 proteins in the parental SGC7901
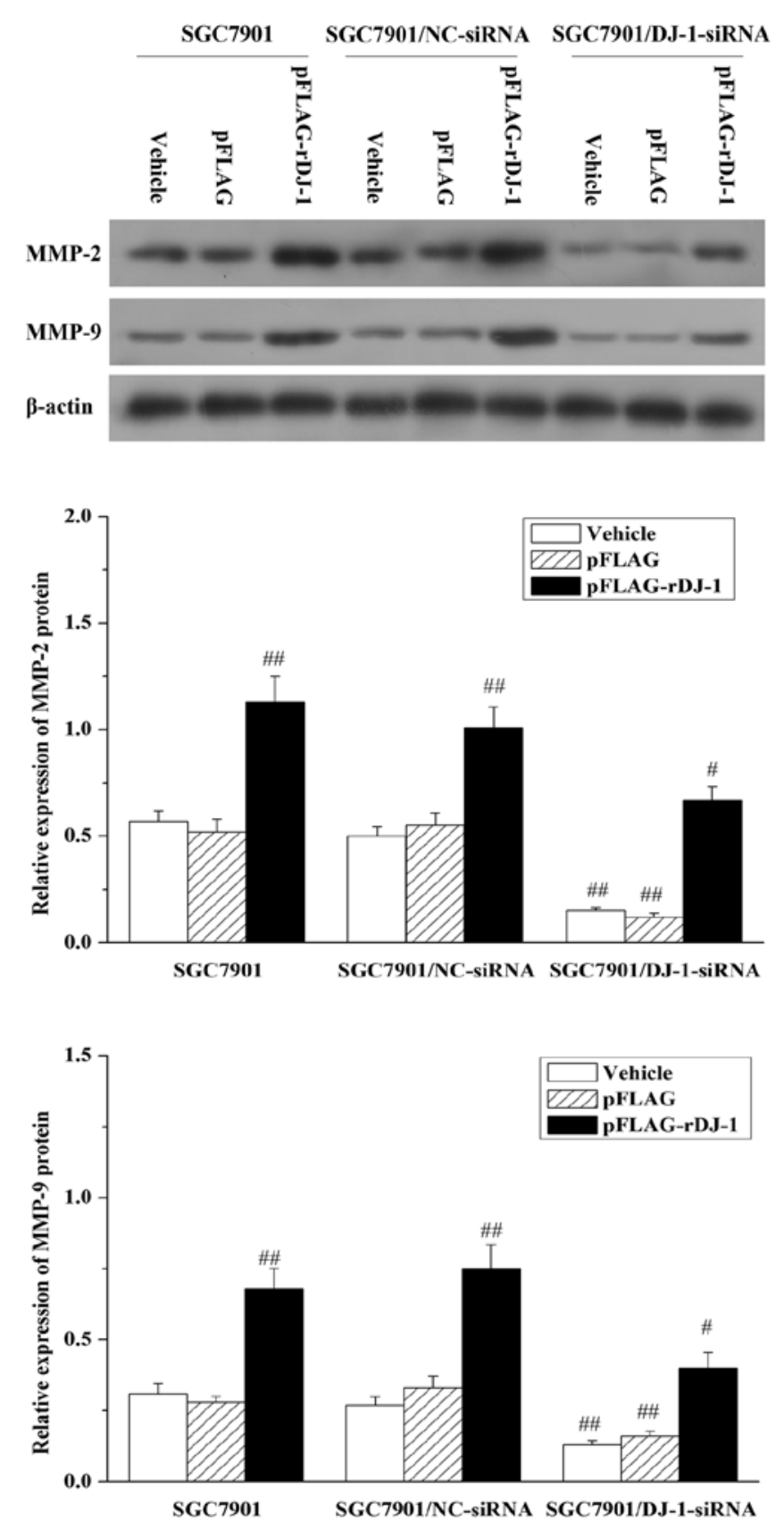

Figure 4. DJ-1 regulates MMP-2 and MMP-9 expression. SGC7901, SGC7901/NC-siRNA and SGC7901/DJ-1-siRNA cells were transfected with or without pFLAG or pFLAG-rDJ-1. Twenty-four hours later, the expression levels of MMP-2 and MMP-9 proteins were analyzed by western blot analysis. $\beta$-actin was used as an internal control. A representative blot of each experiment is shown with the densitometric analysis corresponding to the mean \pm SD of four independent experiments. ${ }^{\#} \mathrm{P}<0.05,{ }^{\# \prime} \mathrm{P}<0.01$ vs. SGC7901 + Vehicle.

and SGC7901/NC-siRNA cells. In contrast, empty vector pFLAG did not affect MMP-2 and MMP-9 expression. These data indicate that DJ-1 can cause the upregulation of MMP-2 and MMP-9 proteins. Subsequently, to study the possible role of MMP-2 and MMP-9 in DJ-1-mediated invasion and migration, SGC7901/DJ-1-siRNA and control cells were treated with $20 \mu \mathrm{M}$ of MMP-2/MMP-9 inhibitor I before performing invasion and migration assays. The results demonstrated that treatment with MMP-2/MMP-9 inhibitor I inhibited the promotive effect of DJ-1 on invasive and migrative abilities 
A

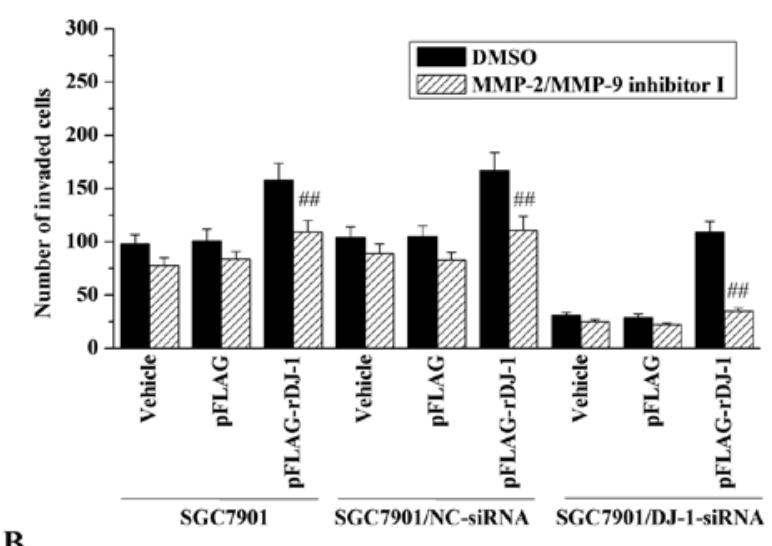

B

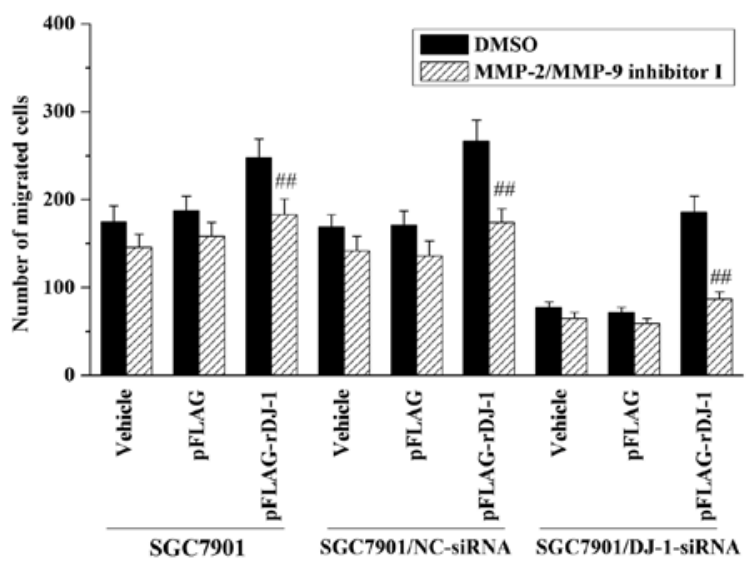

Figure 5. MMP-2/MMP-9 inhibitor I blocks the DJ-1-induced invasion and migration of SGC7901 cells. SGC7901, SGC7901/NC-siRNA and SGC7901/DJ-1-siRNA cells were transfected with or without pFLAG or pFLAG-rDJ-1. Twenty-four hours later, cells were treated for $2 \mathrm{~h}$ with $5 \mu \mathrm{M}$ MMP-2/MMP-9 inhibitor I or 0.1\% (v:v) DMSO as vehicle control, and then (A) cell invasion and (B) migration were respectively analyzed, as described in Materials and methods. Each value represents the mean $\pm \mathrm{SD}$ of four independent experiments. ${ }^{\#} \mathrm{P}<0.05$, ${ }^{\# \#} \mathrm{P}<0.01$ vs. the corresponding DMSO control group.

of the SGC7901 cells (Fig. 5). In addition, MMP-2/MMP-9 inhibitor I also reversed the effects of restored DJ-1 on cell invasion (Fig. 5A) and migration (Fig. 5B) in DJ-1-knockdown cells. Taken together, these data indicate that MMP-2 and MMP-9 may be involved in the promotive effects of DJ-1 on invasion and migration of gastric cancer cells.

The Akt pathway is involved in the upregulation of MMP-2 and $M M P-9$ and the invasion and migration of gastric cancer cells mediated by DJ-1. It has been reported that DJ-1 induces the Akt pathway by negatively regulating the function of the tumorsuppressor gene phosphatase and tensin homolog (PTEN) (30), whereas the Akt pathway has been shown to play a critical role in the migration and invasion of many types of cancer cells $(31,32)$. Thus, we next examined whether the Akt pathway might also be involved in DJ-1-related migration and invasion of gastric cancer cells. Expectedly, in SGC7901/DJ-1-siRNA cells, knockdown of DJ-1 resulted in downregulation of phosphorylated Akt (Thr308). Likewise, the effect was also reversed by restoration of DJ-1 expression and was increased further by exogenous expression of DJ-1 in the parental SGC7901 and SGC7901/

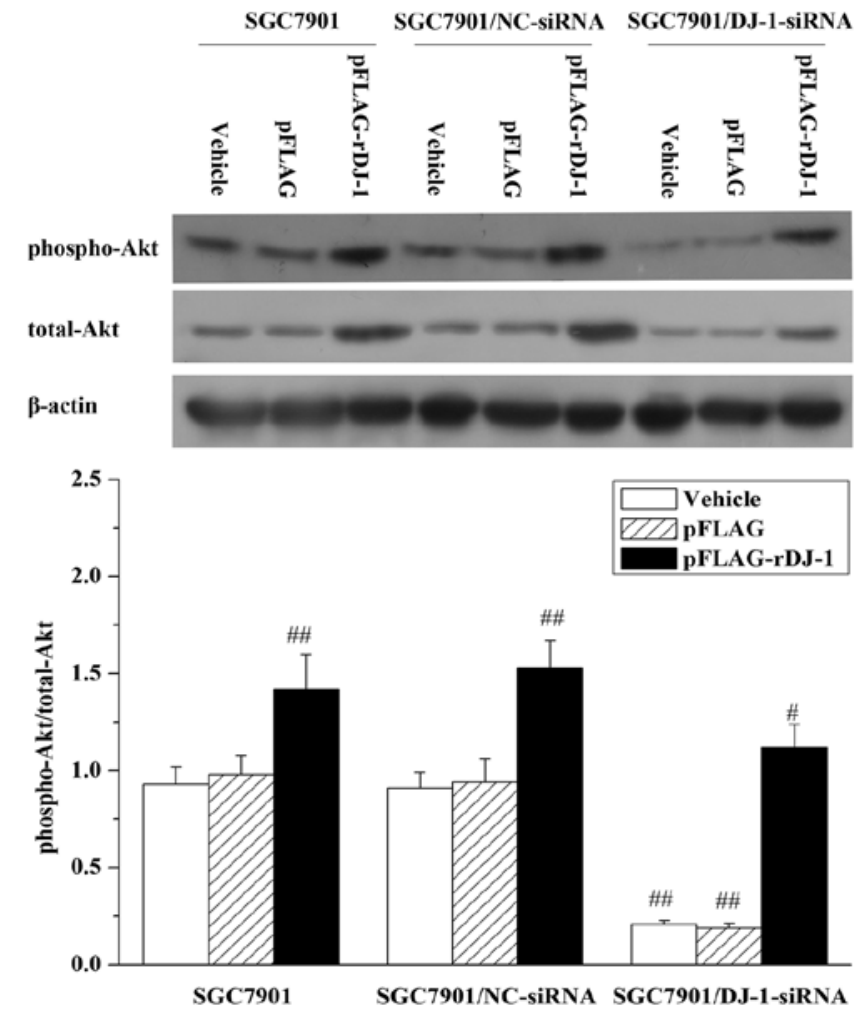

Figure 6. DJ-1 regulates the phosphorylation of Akt. SGC7901, SGC7901/NC-siRNA and SGC7901/DJ-1-siRNA cells were transfected with or without pFLAG or pFLAG-rDJ-1. Twenty-four hours later, the expression of phospho-Akt and total-Akt proteins was analyzed by western blot analysis. $\beta$-actin was used as an internal control. A representative blot of each experiment is shown with the densitometric analysis corresponding to the mean $\pm \mathrm{SD}$ of four independent experiments. ${ }^{\#} \mathrm{P}<0.05,{ }^{\# \#} \mathrm{P}<0.01$ vs. SGC7901 + Vehicle.

NC-siRNA cells (Fig. 6). To further determine whether the promotive effects of DJ-1 on cell invasion and migration were dependent on the Akt pathway, cell invasion and migration were assessed following inhibition of Akt by API-2 in parental SGC7901 cells. As shown in Fig. 7, inhibition of Akt had the same effects as knockdown of DJ-1, with a decrease in MMP-2 and MMP-9 expression (Fig. 7A), a reduction in gastric cancer cell invasion (Fig. 7B) and migration potential (Fig. 7C) in the parental SGC7901 cells. In addition, inhibition of Akt by API-2 reversed the effects of restored DJ-1 on MMP-2 and MMP-9 upregulation (Fig. 8A), invasion (Fig. 8B) and cell migration (Fig. 8C) in DJ-1 knockdown cells. All of these results suggest that the Akt pathway is involved in DJ-1-related invasion and migration, and is mediated by the upregulation of MMP-2 and MMP-9 expression induced by DJ-1.

\section{Discussion}

It is known that the majority of gastric cancer deaths result from peritoneal metastases rather than from primary tumors. Yet, the molecular mechanisms regulating peritoneal metastasis of gastric cancer remain incompletely defined. DJ-1, a ubiquitously expressed and highly conserved intracellular protein, was discovered as a mitogen-dependent oncogene product by Nagakubo et al (6). More recently, DJ-1 has been reported to play an important role during the invasion and metastasis of many types of carcinoma cells, including uveal malignant 

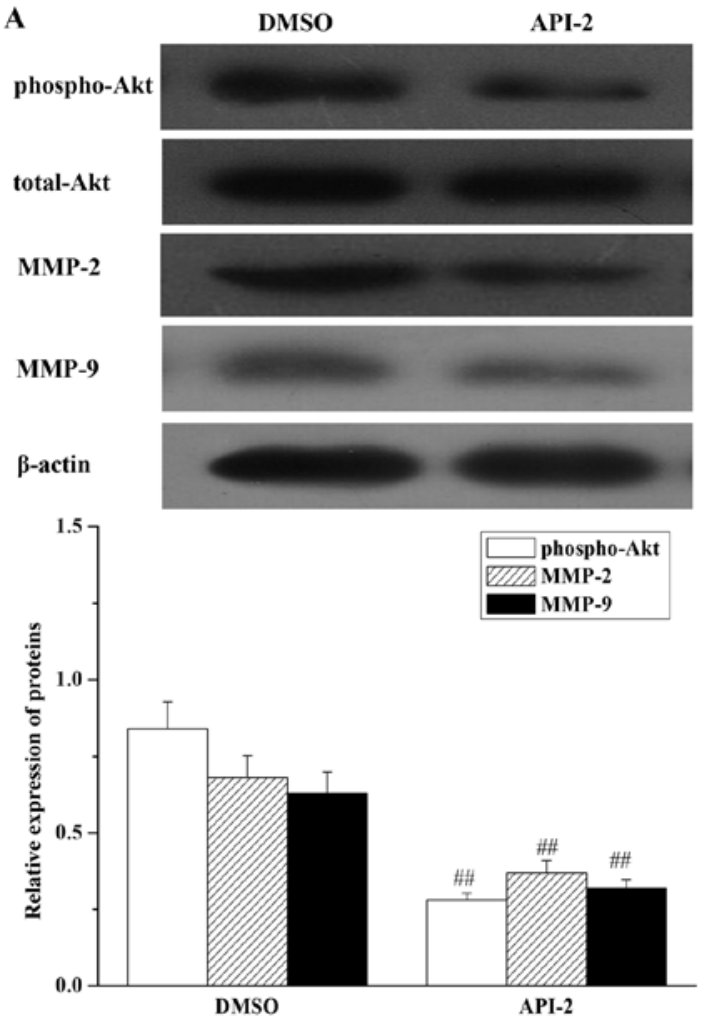
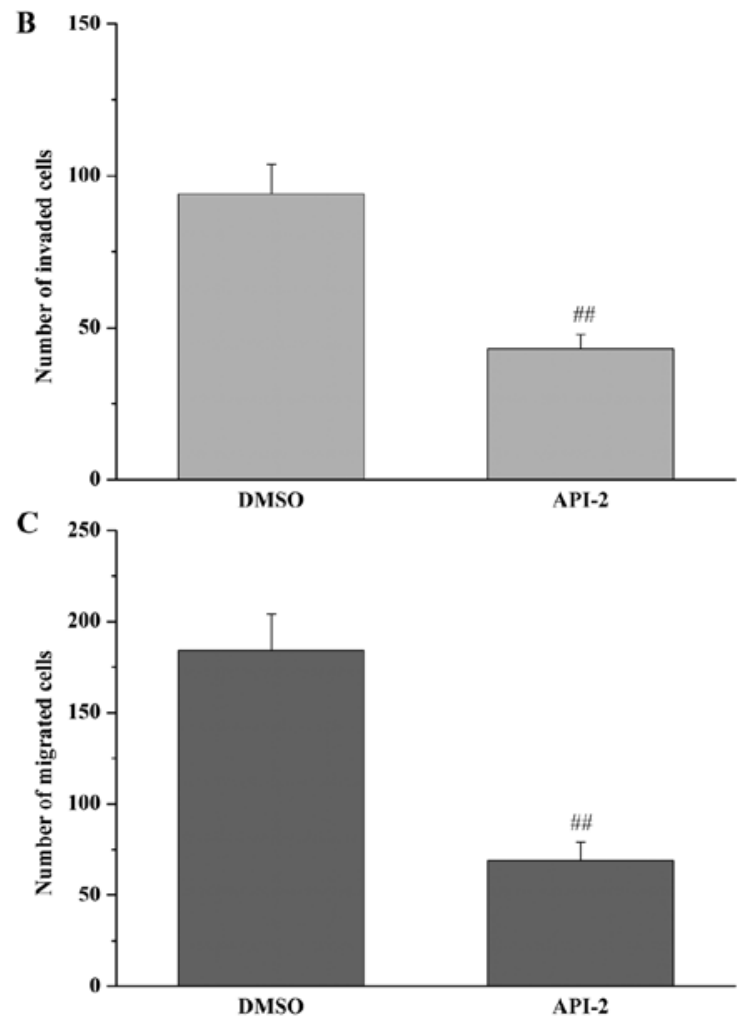

Figure 7. Inhibition of Akt attenuates cell migration and invasion, and the expression of MMP-2 and MMP-9 proteins in SGC7901 cells. SGC7901 cells were treated for $24 \mathrm{~h}$ with $10 \mu \mathrm{M}$ API-2 or $0.1 \%$ (v:v) DMSO as vehicle control, and (A) expression of phospho-Akt, total-Akt, MMP-2 and MMP-9, and (B) cell invasion and (C) migration were, respectively, analyzed, as described in Materials and methods. Each value represents the mean \pm SD of four independent experiments. ${ }^{\#} \mathrm{P}<0.01$ vs. the corresponding DMSO control group.

melanoma (19), esophageal squamous cell carcinoma (20), primary lung cancer (21), glioma (22) and pancreatic cancer $(18,23)$. However, to date, there are no reports concerning the role of DJ-1 in the peritoneal metastasis of gastric cancer.

In the present study, we presented initial evidence that the expression of DJ-1 is significantly upregulated in gastric cancers with peritoneal metastasis compared to that in cancers without peritoneal metastasis or normal gastric mucosa. We also found that DJ-1 was overexpressed in gastric cancer cells that had a high degree of metastatic potential, as compared with corresponding normal cells. These results indicate that the overexpression of DJ-1 may be closely related to the development of peritoneal metastasis by gastric carcinoma. However, whether there is a causal relationship between DJ-1 expression and peritoneal metastasis of gastric carcinoma deserves further exploration. Thus, in the present study, we generated stable transfectants to knockdown DJ-1 in SGC7901 cells and further tested the ability of DJ-1-knockdown cells to form tumor nodules in the peritoneal cavity using an in vivo model of peritoneal metastasis in nude mice. The results showed that DJ-1-knockdown SGC7901 cells had a decreased ability to form peritoneal metastatic nodules when compared to the control cells (parental SGC7901 and SGC7901/NC-siRNA cells). This suggests that DJ-1 is involved in the development of peritoneal metastasis by gastric carcinoma.

The ability to migrate and invade the basement membrane into surrounding tissues, blood and lymphatic vessels is one of the essential hallmarks of cancer and is a prerequisite for local tumor progression and metastatic spread (33). Accordingly, to identify the mechanisms regulating the decreased ability of DJ-1-knockdown cells to form peritoneal metastasis, the migration and invasion capacities in vitro were also analyzed. As expected, we observed that DJ-1-knockdown SGC7901 cells exhibited a marked decrease in both cell invasion and migration, which was reversed by restoration of DJ-1 expression, and was increased further by ectopic expression of DJ-1 in parental SGC7901 cells. Our data indicate that DJ-1 regulates gastric cancer cell migration and invasion properties in cell culture. Extensive studies have shown that DJ-1 plays an important role in cell proliferation and survival $(30,34,35)$; however, our in vitro assay indicated that cell proliferation and survival remained unchanged upon knockdown of DJ-1 expression (data not shown). This suggests that the ability of DJ-1 to promote peritoneal metastasis is mainly due to its effects on the regulation of gastric cancer cell migration and invasion, independent of cellular proliferation. The essential requirement for DJ-1 in gastric cancer cell migration and invasion highlights the potential for using DJ-1 as a target for blocking gastric cancer metastasis.

Our studies also indicate the mechanisms by which DJ-1 modulates cell migration and invasion. It is known that cancer cell invasion and metastasis require controlled degradation of the extracellular matrix. Matrix metalloproteinases (MMPs) are a family of zinc-dependent endopeptidases which play an important role in the proteolytic destruction of extracellular matrix and basement membranes, thereby, they are essential for tumor invasion and metastasis (36). MMPs, particularly MMP-2 and MMP-9 have been implicated in cancer invasion 
A
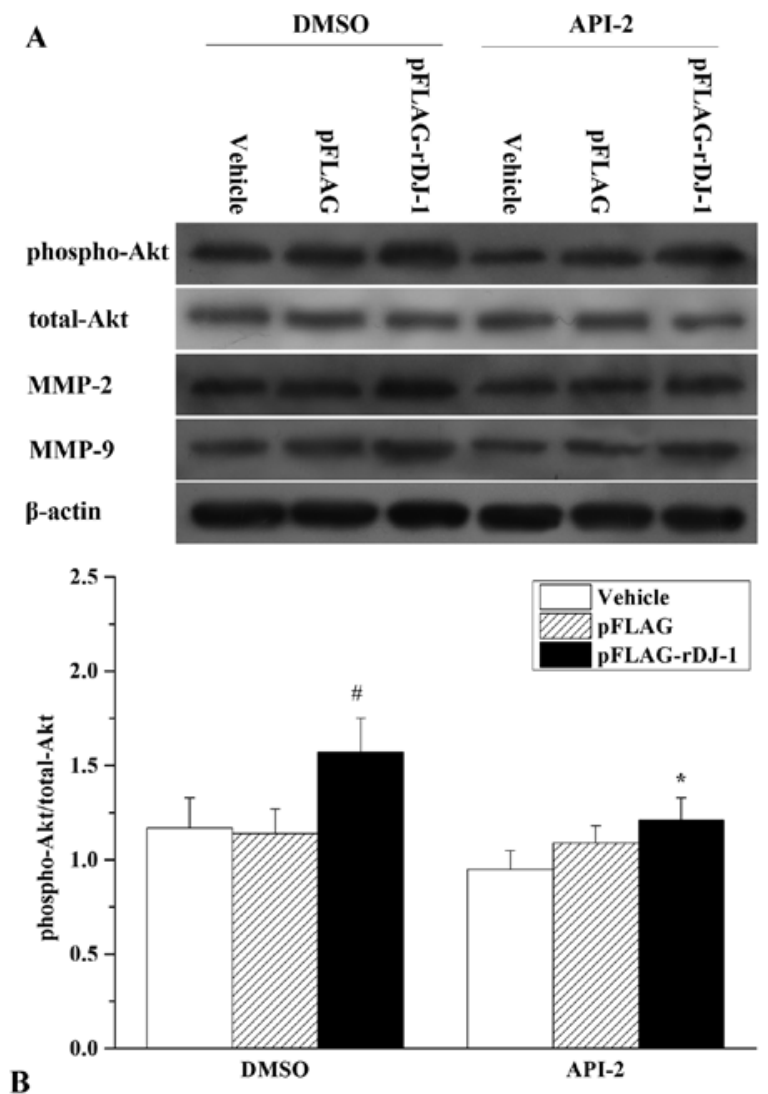

B
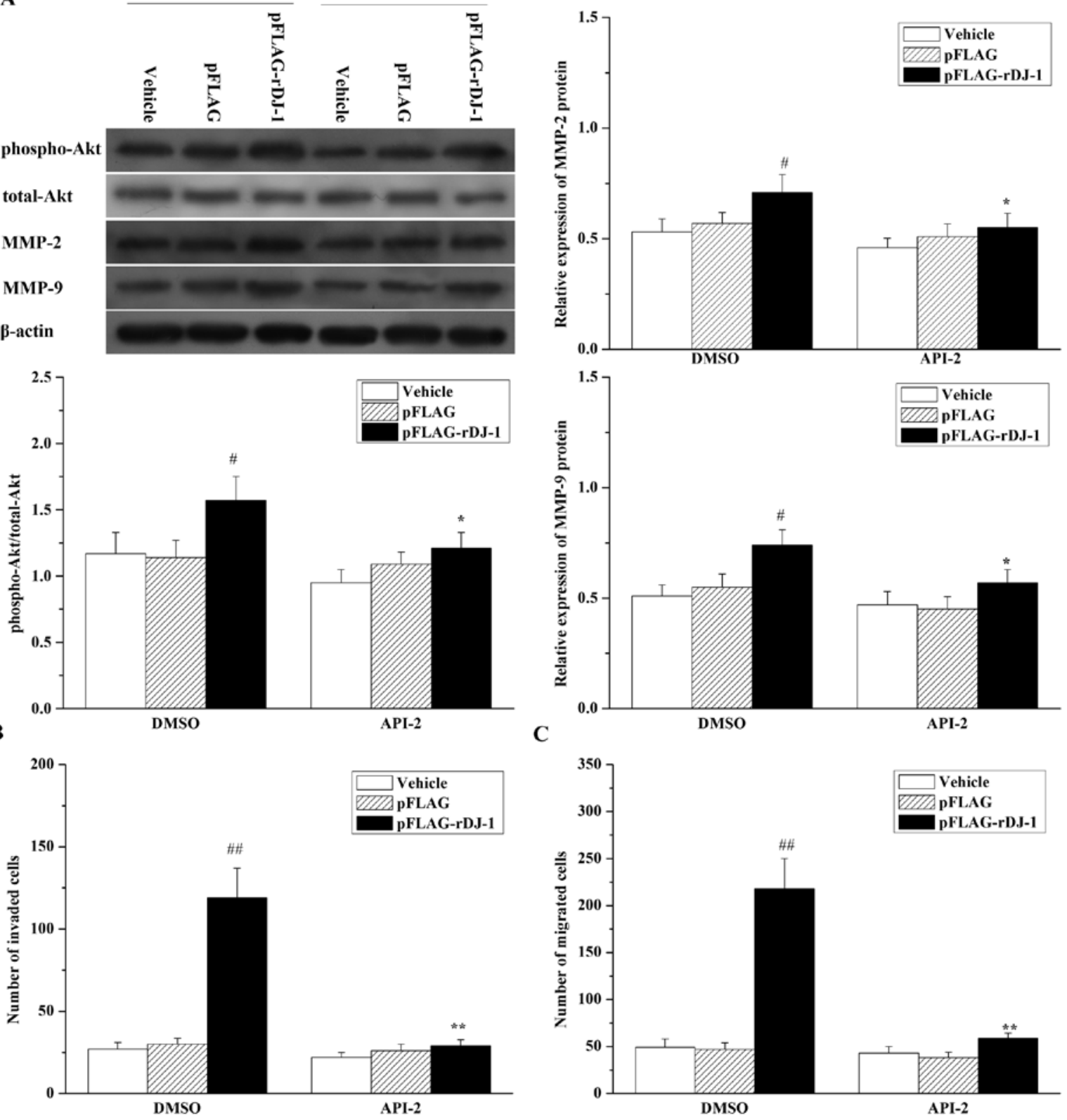

C

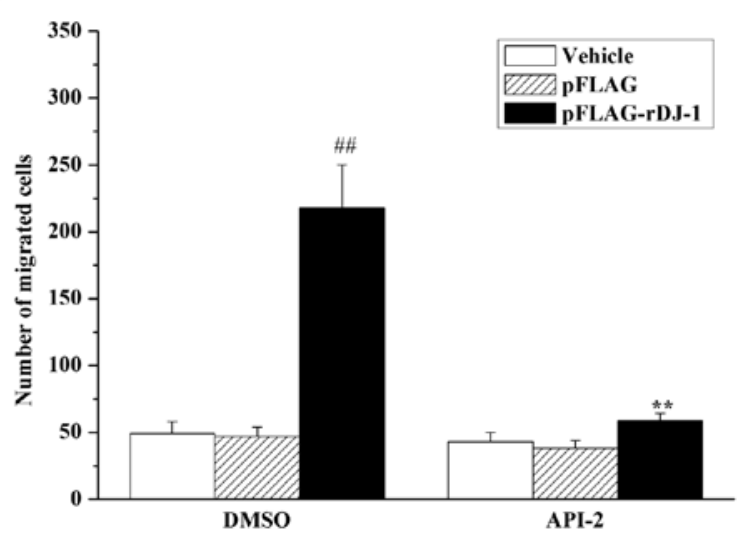

Figure 8. Inhibition of Akt reverses the effects of restored DJ-1 on cell migration and invasion, and the expression of MMP-2 and MMP-9 proteins in SGC7901/DJ-1-siRNA cells. SGC7901/DJ-1-siRNA cells were transfected with or without pFLAG or pFLAG-rDJ-1. Twenty-four hours later, cells were treated for $24 \mathrm{~h}$ with $20 \mu \mathrm{M}$ API-2 or 0.1\% (v:v) DMSO as vehicle control, and (A) expression of phospho-Akt, total-Akt, MMP-2 and MMP-9, and (B) cell invasion and $(\mathrm{C})$ migration were, respectively, analyzed, as described in Materials and methods. Each value represents the mean $\pm \mathrm{SD}$ of four independent experiments. ${ }^{~} \mathrm{P}<0.05,{ }^{\# \#} \mathrm{P}<0.01$ vs. DMSO + Vehicle ${ }^{*} \mathrm{P}<0.05,{ }^{* *} \mathrm{P}<0.01$ vs. DMSO + pFLAG-rDJ-1.

and metastasis (29). Thus, it is necessary to investigate whether the involvement of DJ-1 in invasion and migration is also correlated with MMP-2 and MMP-9 in gastric cancer. Notably, we found that, in SGC7901/DJ-1-siRNA cells, DJ-1 silencing resulted in reduction in MMP-2 and MMP-9 expression, which was also reversed by restoration of DJ-1 expression. Moreover, blockage of MMP-2 and MMP-9 activities by MMP-2/MMP-9 inhibitor I reversed the promotive effect of DJ-1 on invasive and migrative abilities in gastric cancer cells. These results indicate that MMP-2 and MMP-9 may be involved in the promotion of cell invasion and migration by DJ-1.

In order to understand the connection between DJ-1 and gastric cancer cell invasion and migration in more detail, iden- tification of the signaling cascades by which DJ-1 regulates invasion and migration is important. It has been reported that DJ-1 induces the Akt pathway by negatively regulating the function of the tumor-suppressor gene PTEN (30). Moreover, the Akt pathway has been shown to play a critical role in the migration and invasion of many types of cancer cells by regulating the expression of multiple metastasis-related genes, including MMP-2 and MMP-9 $(31,32)$. Thus, we speculated that the Akt pathway might also be responsible for the DJ-1mediated migration and invasion of gastric cancer cells. To verify this hypothesis, we investigated the phosphorylated status of Akt and its correlation with MMP-2 and MMP-9 expression and cell migration and invasion in SGC7901 cells. 
Our results demonstrated that downregulation of DJ-1 caused a marked decrease in Akt phosphorylation, with concomitant down-regulation of MMP-2 and MMP-9 expression and inhibition of cell invasion and migration in SGC7901/DJ-1-siRNA cells. In addition, we demonstrated that inhibition of the Akt pathway by API-2 mimicked the effects of DJ-1 silencing in parental SGC7901 and reversed the effects of restored DJ-1 on MMP-2 and MMP-9 expression, cell migration and invasion. All of these results suggest that the Akt pathway is specifically involved in DJ-1-related invasion and migration, and mediates the upregulation of MMP-2 and MMP-9 expression induced by DJ-1. However, in the peritoneal metastatic of gastric cancer, the means by which DJ-1 activates the Akt pathway remains unknown and warrants further study.

In summary, the present study provides unequivocal evidence that DJ-1 is correlated with peritoneal metastatic of gastric cancer and the promotion of cell invasion and migration. The effects are at least partially mediated by activation of the Akt pathway and consequent upregulation of MMP-2 and MMP-9 expression. This suggests that DJ-1 may be a potential therapeutic target for blocking peritoneal carcinomatosis of gastric carcinoma.

\section{Acknowledgements}

The present study was supported by the Natural Scientific Foundation of China (no. 81160304), the Scientific Research Foundation of Jiangxi Provincial Educational Department (no. GJJ12062), and the Training Program for Young Scientists of Jiangxi Province (no. 20133BCB23028).

\section{References}

1. Yonemura Y, Endou Y, Sasaki T, et al: Surgical treatment for peritoneal carcinomatosis from gastric cancer. Eur J Surg Oncol 36: 1131-1138, 2010

2. Takahashi I, Matsusaka T, Onohara T, et al: Clinicopathological features of long-term survivors of scirrhous gastric cancer. Hepatogastroenterology 47: 1485-1488, 2000.

3. Fujimura T, Ishii K, Oyama K, et al: A new scoring system for peritoneal metastasis in gastric cancer. Gastric Cancer 6: 146-152, 2003

4. Bando E, Yonemura Y, Takeshita Y, et al: Intraoperative lavage for cytological examination in 1,297 patients with gastric carcinoma. Am J Surg 178: 256-262, 1999.

5. Hippo Y, Yashiro M, Ishii M, et al: Differential gene expression profiles of scirrhous gastric cancer cells with high metastatic potential to peritoneum or lymph nodes. Cancer Res 61: 889-895, 2001.

6. Nagakubo D, Taira T, Kitaura H, et al: DJ-1, a novel oncogene which transforms mouse NIH3T3 cells in cooperation with ras. Biochem Biophys Res Commun 231: 509-513, 1997.

7. Ishikawa S, Taira T, Takahashi-Niki K, Niki T, Ariga H and Iguchi-Ariga SM: Human DJ-1-specific transcriptional activation of tyrosine hydroxylase gene. J Biol Chem 285: 39718-39731, 2010.

8. Shinbo Y, Taira T, Niki T, Iguchi-Ariga SM and Ariga H: DJ-1 restores $\mathrm{p} 53$ transcription activity inhibited by Topors/p53BP3. Int J Oncol 26: 641-648, 2005.

9. Taira T, Saito Y, Niki T, Iguchi-Ariga SM, Takahashi K and Ariga H: DJ-1 has a role in antioxidative stress to prevent cell death. EMBO Rep 5: 213-218, 2004

10. Shendelman S, Jonason A, Martinat C, Leete T and Abeliovich A: DJ-1 is a redox-dependent molecular chaperone that inhibits a-synuclein aggregate formation. PLoS Biol 2: e362, 2004.

11. Honbou K, Suzuki NN, Horiuchi M, et al: The crystal structure of DJ-1, a protein related to male fertility and Parkinson's disease. J Biol Chem 278: 31380-31384, 2003.
12. Le Naour F, Misek DE, Krause MC, et al: Proteomics-based identification of RS/DJ-1 as a novel circulating tumor antigen in breast cancer. Clin Cancer Res 7: 3328-3335, 2001.

13. MacKeigan JP, Clements CM, Lich JD, Pope RM, Hod Y and Ting JP: Proteomic profiling drug-induced apoptosis in non-small cell lung carcinoma: identification of RS/DJ-1 and RhoGDIa. Cancer Res 63: 6928-6934, 2003.

14. Liu H, Wang M, Li M, et al: Expression and role of DJ-1 in leukemia. Biochem Biophys Res Commun 375: 477-483, 2008.

15. Hod Y: Differential control of apoptosis by DJ-1 in prostate benign and cancer cells. J Cell Biochem 92: 1221-1233, 2004.

16. Arnouk H, Merkley MA, Podolsky RH, et al: Characterization of molecular markers indicative of cervical cancer progression. Proteomics Clin Appl 3: 516-527, 2009.

17. Giusti L, Iacconi P, Ciregia F, et al: Fine-needle aspiration of thyroid nodules: proteomic analysis to identify cancer biomarkers. J Proteome Res 7: 4079-4088, 2008.

18. Tian M, Cui YZ, Song GH, et al: Proteomic analysis identifies MMP-9, DJ-1 and A1BG as overexpressed proteins in pancreatic juice from pancreatic ductal adenocarcinoma patients. BMC Cancer 8: 241, 2008.

19. Pardo M, Garcia A, Thomas B, et al: The characterization of the invasion phenotype of uveal melanoma tumour cells shows the presence of MUC18 and HMG-1 metastasis markers and leads to the identification of DJ-1 as a potential serum biomarker. Int J Cancer 119: 1014-1022, 2006.

20. Yuen HF, Chan YP, Law S, et al: DJ-1 could predict worse prognosis in esophageal squamous cell carcinoma. Cancer Epidemiol Biomarkers Prev 17: 3593-3602, 2008.

21. Bai J, Guo C, Sun W, et al: DJ-1 may contribute to metastasis of non-small cell lung cancer. Mol Biol Rep 39: 2697-2703, 2012.

22. Fang M, Zhong XY, Du B, et al: Role of DJ-1-induced PTEN down-regulation in migration and invasion of human glioma cells. Chin J Cancer 29: 988-994, 2010.

23. He X, Zheng Z, Li J, et al: DJ-1 promotes invasion and metastasis of pancreatic cancer cells by activating SRC/ERK/uPA. Carcinogenesis 33: 555-562, 2012.

24. Wang JW, Peng SY, Li JT, et al: Identification of metastasisassociated proteins involved in gallbladder carcinoma metastasis by proteomic analysis and functional exploration of chloride intracellular channel 1. Cancer Lett 281: 71-81, 2009.

25. Li Z, Zhan W, Wang Z, et al: Inhibition of PRL-3 gene expression in gastric cancer cell line SGC7901 via microRNA suppressed reduces peritoneal metastasis. Biochem Biophys Res Commun 348: 229-237, 2006.

26. Yasumoto K, Koizumi K, Kawashima A, et al: Role of the CXCL12/CXCR4 axis in peritoneal carcinomatosis of gastric cancer. Cancer Res 66: 2181-2187, 2006.

27. Sako A, Kitayama J, Koyama H, et al: Transduction of soluble Flt-1 gene to peritoneal mesothelial cells can effectively suppress peritoneal metastasis of gastric cancer. Cancer Res 64: 3624-3628, 2004.

28. Pan Y, Zhao L, Liang J, et al: Cellular prion protein promotes invasion and metastasis of gastric cancer. FASEB J 20: 1886-1888, 2006.

29. Khasigov PZ, Podobed OV, Gracheva TS, Salbiev KD, Grachev SV and Berezov TT: Role of matrix metalloproteinases and their inhibitors in tumor invasion and metastasis. Biochemistry (Mosc) 68: 711-717, 2003.

30. Kim RH, Peters M, Jang Y, et al: DJ-1, a novel regulator of the tumor suppressor PTEN. Cancer Cell 7: 263-273, 2005.

31. Yoeli-Lerner M and Toker A: Akt/PKB signaling in cancer: a function in cell motility and invasion. Cell Cycle 5: 603-605, 2006.

32. Kim D, Kim S, Koh H, et al: Akt/PKB promotes cancer cell invasion via increased motility and metalloproteinase production. FASEB J 15: 1953-1962, 2001.

33. Geiger TR and Peeper DS: Metastasis mechanisms. Biochim Biophys Acta 1796: 293-308, 2009.

34. Aron L, Klein P, Pham TT, Kramer ER, Wurst W and Klein R: Pro-survival role for Parkinson's associated gene DJ-1 revealed in trophically impaired dopaminergic neurons. PLoS Biol 8: e1000349, 2010.

35. Vasseur S, Afzal S, Tardivel-Lacombe J, Park DS, Iovanna JL and Mak TW: DJ-1/PARK7 is an important mediator of hypoxiainduced cellular responses. Proc Natl Acad Sci USA 106: 1111-1116, 2009.

36. Nabeshima K, Inoue T, Shimao $\mathrm{Y}$ and Sameshima T: Matrix metalloproteinases in tumor invasion: role for cell migration. Pathol Int 52: 255-264, 2002. 